\title{
Families of rational solutions of order 5 to the KPI equation depending on 8 parameters.
}

\author{
Pierre Gaillard \\ Institut de Mathématiques, Université de Bourgogne, Dijon, France \\ Email: Pierre.Gaillard@u-bourgogne.fr
}

\begin{abstract}
In this paper, we go on with the study of rational solutions to the Kadomtsev-Petviashvili equation (KPI). We construct here rational solutions of order 5 as a quotient of 2 polynomials of degree 60 in $x, y$ and $t$ depending on 8 parameters. The maximum modulus of these solutions at order 5 is checked as equal to $2(2 N+1)^{2}=242$. We study their modulus patterns in the plane $(x, y)$ and their evolution according to time and parameters $a_{1}, a_{2}, a_{3}, a_{4}, b_{1}, b_{2}, b_{3}, b_{4}$. We get triangle and ring structures as obtained in the case of the NLS equation.
\end{abstract}

Keywords: Kadomtsev petviashvili equation, rogue waves, lumps.

\section{Introduction}

We consider the Kadomtsev-Petviashvili equation (KPI) in the following form

$$
\left(4 u_{t}-6 u u_{x}+u_{x x x}\right)_{x}-3 u_{y y}=0,
$$

where subscripts $x, y$ and $t$ denote partial derivatives.

Kadomtsev and Petviashvili [1] first proposed this equation in 1970. This equation is a model for example, for surface and internal water waves [2], and in nonlinear optics [3]. Zakharov extended the inverse scattering transform (IST) to this KPI equation, and obtained several exact solutions.

The first rational solutions were found in 1977 by Manakov, Zakharov, Bordag and Matveev [4]. Other researches were led and more general rational solutions to the KPI equation were obtained. We can mention the following works by Krichever in 1978 [5], [6], Satsuma and Ablowitz in 1979 [7], Matveev in 1979 [8], Freeman and Nimmo in 1983 [9], [10], Pelinovsky and Stepanyants in 1993 [11], Pelinovsky in 1994 [12], Ablowitz and Villarroel [13], [14] in 1997-1999, Biondini and Kodama [15], [16], [17] in 2003-2007.

We recall the author's results about the representations of the solutions to the KPI equation, first in terms of Fredholm determinants of order $2 N$ depending on $2 N-1$ parameters, then in terms of wronskians of order $2 N$ with $2 N-1$ parameters. These representations allow to obtain an infinite hierarchy of solutions to the KPI equation, depending on $2 N-1$ real parameters .

Then we construct the rational solutions of order $N$ depending on $2 N-2$ parameters without presence of a limit which can be written as a ratio of two polynomials of $x, y$ and $t$ of degree $2 N(N+1)$.

The maximum modulus of these solutions at order $N$ is equal to $2(2 N+1)^{2}$. This method gives an infinite hierarchy of rational solutions of order $N$ depending on $2 N-2$ real parameters. We construct here the explicit rational solutions of order 5 , depending on 8 real parameters, and the representations of their modulus in the plane of the coordinates $(x, y)$ according to the real parameters $a_{1}, b_{1}, a_{2}, b_{2}, a_{3}, b_{3}, a_{4}$, $b_{4}$ and time $t$. 


\section{Rational Solutions to the KPI equation of order $N$ depending on $2 N-2$ parameters}

\subsection{Fredholm Representation}

One defines real numbers $\lambda_{j}$ such that $-1<\lambda_{\nu}<1, \nu=1, \ldots, 2 N$ depending on a parameter $\epsilon$ that will be intended to tend towards 0 ; they can be written as

$$
\lambda_{j}=1-2 \epsilon^{2} j^{2}, \quad \lambda_{N+j}=-\lambda_{j}, \quad 1 \leq j \leq N,
$$

The terms $\kappa_{\nu}, \delta_{\nu}, \gamma_{\nu}, \tau_{\nu}$ and $x_{r, \nu}$ are functions of $\lambda_{\nu}, 1 \leq \nu \leq 2 N$; they are defined by the formulas :

$$
\begin{aligned}
& \kappa_{j}=2 \sqrt{1-\lambda_{j}^{2}}, \quad \delta_{j}=\kappa_{j} \lambda_{j}, \quad \gamma_{j}=\sqrt{\frac{1-\lambda_{j}}{1+\lambda_{j}}} ; \\
& x_{r, j}=(r-1) \ln \frac{\gamma_{j}-i}{\gamma_{j}+i}, \quad r=1,3, \quad \tau_{j}=-12 i \lambda_{j}^{2} \sqrt{1-\lambda_{j}^{2}}-4 i\left(1-\lambda_{j}^{2}\right) \sqrt{1-\lambda_{j}^{2}}, \\
& \kappa_{N+j}=\kappa_{j}, \quad \delta_{N+j}=-\delta_{j}, \quad \gamma_{N+j}=\gamma_{j}^{-1}, \\
& x_{r, N+j}=-x_{r, j}, \quad \tau_{N+j}=\tau_{j} \quad j=1, \ldots, N .
\end{aligned}
$$

$e_{\nu} 1 \leq \nu \leq 2 N$ are defined in the following way:

$$
\begin{aligned}
& e_{j}=2 i\left(\sum_{k=1}^{1 / 2 M-1} a_{k}(j e)^{2 k-1}-i \sum_{k=1}^{1 / 2 M-1} b_{K}(j e)^{2 k-1}\right), \\
& e_{N+j}=2 i\left(\sum_{k=1}^{1 / 2 M-1} a_{k}(j e)^{2 k-1}+i \sum_{k=1}^{1 / 2 M-1} b_{k}(j e)^{2 k-1}\right), \quad 1 \leq j \leq N, \\
& a_{k}, b_{k} \in \mathbf{R}, \quad 1 \leq k \leq N-1 .
\end{aligned}
$$

$\epsilon_{\nu}, 1 \leq \nu \leq 2 N$ are real numbers defined by :

$$
e_{j}=1, \quad e_{N+j}=0 \quad 1 \leq j \leq N .
$$

Let $I$ be the unit matrix and $D_{r}=\left(d_{j k}\right)_{1 \leq j, k \leq 2 N}$ the matrix defined by :

$$
d_{\nu \mu}=(-1)^{\epsilon_{\nu}} \prod_{\eta \neq \mu}\left(\frac{\gamma_{\eta}+\gamma_{\nu}}{\gamma_{\eta}-\gamma_{\mu}}\right) \exp \left(i \kappa_{\nu} x-2 \delta_{\nu} y+\tau_{\nu} t+x_{r, \nu}+e_{\nu}\right) .
$$

Then we recall the following result :

Theorem 2.1 The function $v$ defined by

$$
v(x, y, t)=-2 \frac{|n(x, y, t)|^{2}}{d(x, y, t)^{2}}
$$

where

$$
\begin{aligned}
& n(x, y, t)=\operatorname{det}\left(I+D_{3}(x, y, t)\right), \\
& d(x, y, t)=\operatorname{det}\left(I+D_{1}(x, y, t)\right),
\end{aligned}
$$

and $D_{r}=\left(d_{j k}\right)_{1 \leq j, k \leq 2 N}$ the matrix

$$
d_{\nu \mu}=(-1)^{\epsilon_{\nu}} \prod_{\eta \neq \mu}\left(\frac{\gamma_{\eta}+\gamma_{\nu}}{\gamma_{\eta}-\gamma_{\mu}}\right) \exp \left(i \kappa_{\nu} x-2 \delta_{\nu} y+\tau_{\nu} t+x_{r, \nu}+e_{\nu}\right) .
$$

is a solution to the KPI equation (1), depending on $2 N-1$ parameters $a_{k}, b_{k}, 1 \leq k \leq N-1$ and $\epsilon$. 


\subsection{Wronskian Representation}

We use the following notations :

$$
\phi_{r, \nu}=\sin \Theta_{r, \nu}, \quad 1 \leq \nu \leq N, \quad \phi_{r, \nu}=\cos \Theta_{r, \nu}, \quad N+1 \leq \nu \leq 2 N, \quad r=1,3,
$$

with the arguments

$$
\Theta_{r, \nu}=\frac{\kappa_{\nu} x}{2}+i \delta_{\nu} y-i \frac{x_{r, \nu}}{2}-i \frac{\tau_{\nu}}{2} t+\gamma_{\nu} w-i \frac{e_{\nu}}{2}, \quad 1 \leq \nu \leq 2 N .
$$

$W_{r}(w)$ denotes the wronskian of the functions $\phi_{r, 1}, \ldots, \phi_{r, 2 N}$ defined by

$$
W_{r}(w)=\operatorname{det}\left[\left(\partial_{w}^{\mu-1} \phi_{r, \nu}\right)_{\nu, \mu \in[1, \ldots, 2 N]}\right]
$$

We consider the matrix $D_{r}=\left(d_{\nu \mu}\right)_{\nu, \mu \in[1, \ldots, 2 N]}$ defined in (10). Then we have the following statement :

Theorem 2.2 The function $v$ defined by :

$$
v(x, y, t)=-2 \frac{\left|W_{3}\left(\phi_{3,1}, \ldots, \phi_{3,2 N}\right)(0)\right|^{2}}{\left(W_{1}\left(\phi_{1,1}, \ldots, \phi_{1,2 N}\right)(0)\right)^{2}}
$$

is a solution to the KPI equation depending on $2 N-1$ real parameters $a_{k}, b_{k} 1 \leq k \leq N-1$ and $\epsilon$, with $\phi_{\nu}^{r}$ defined in (11)

$$
\begin{array}{ll}
\phi_{r, \nu}(w)=\sin \left(\frac{\kappa_{\nu} x}{2}+i \delta_{\nu} y-i \frac{x_{r, \nu}}{2}-i \frac{\tau_{\nu}}{2} t+\gamma_{\nu} w-i \frac{e_{\nu}}{2}\right), & 1 \leq \nu \leq N, \\
\phi_{r, \nu}(w)=\cos \left(\frac{\kappa_{\nu} x}{2}+i \delta_{\nu} y-i \frac{x_{r, \nu}}{2}-i \frac{\tau_{\nu}}{2} t+\gamma_{\nu} w-i \frac{e_{\nu}}{2}\right), & N+1 \leq \nu \leq 2 N, \quad r=1,3,
\end{array}
$$

$\kappa_{\nu}, \delta_{\nu}, x_{r, \nu}, \gamma_{\nu}, e_{\nu}$ being defined in(3), (2) and (4).

\subsection{Rational Solutions}

We recall the last result concerning the rational solutions to the KPI equation as a quotient of two determinants.

We use the following notations :

$$
\begin{aligned}
& X_{\nu}=\frac{\kappa_{\nu} x}{2}+i \delta_{\nu} y-i \frac{x_{3, \nu}}{2}-i \frac{\tau_{\nu} t}{2}-i \frac{e_{\nu}}{2}, \\
& Y_{\nu}=\frac{\kappa_{\nu} x}{2}+i \delta_{\nu} y-i \frac{x_{1, \nu}}{2}-i \frac{\tau_{\nu} t}{2}-i \frac{e_{\nu}}{2},
\end{aligned}
$$

for $1 \leq \nu \leq 2 N$, with $\kappa_{\nu}, \delta_{\nu}, x_{r, \nu}$ defined in (3) and parameters $e_{\nu}$ defined by (4).

We define the following functions :

$$
\begin{aligned}
& \varphi_{4 j+1, k}=\gamma_{k}^{4 j-1} \sin X_{k}, \quad \varphi_{4 j+2, k}=\gamma_{k}^{4 j} \cos X_{k}, \\
& \varphi_{4 j+3, k}=-\gamma_{k}^{4 j+1} \sin X_{k}, \quad \varphi_{4 j+4, k}=-\gamma_{k}^{4 j+2} \cos X_{k},
\end{aligned}
$$

for $1 \leq k \leq N$, and

$$
\begin{aligned}
& \varphi_{4 j+1, N+k}=\gamma_{k}^{2 N-4 j-2} \cos X_{N+k}, \quad \varphi_{4 j+2, N+k}=-\gamma_{k}^{2 N-4 j-3} \sin X_{N+k}, \\
& \varphi_{4 j+3, N+k}=-\gamma_{k}^{2 N-4 j-4} \cos X_{N+k}, \quad \varphi_{4 j+4, N+k}=\gamma_{k}^{2 N-4 j-5} \sin X_{N+k},
\end{aligned}
$$

for $1 \leq k \leq N$.

We define the functions $\psi_{j, k}$ for $1 \leq j \leq 2 N, 1 \leq k \leq 2 N$ in the same way, the term $X_{k}$ is only replaced by $Y_{k}$.

$$
\begin{aligned}
& \psi_{4 j+1, k}=\gamma_{k}^{4 j-1} \sin Y_{k}, \quad \psi_{4 j+2, k}=\gamma_{k}^{4 j} \cos Y_{k}, \\
& \psi_{4 j+3, k}=-\gamma_{k}^{4 j+1} \sin Y_{k}, \quad \psi_{4 j+4, k}=-\gamma_{k}^{4 j+2} \cos Y_{k},
\end{aligned}
$$


for $1 \leq k \leq N$, and

$$
\begin{aligned}
& \psi_{4 j+1, N+k}=\gamma_{k}^{2 N-4 j-2} \cos Y_{N+k}, \quad \psi_{4 j+2, N+k}=-\gamma_{k}^{2 N-4 j-3} \sin Y_{N+k}, \\
& \psi_{4 j+3, N+k}=-\gamma_{k}^{2 N-4 j-4} \cos Y_{N+k}, \quad \psi_{4 j+4, N+k}=\gamma_{k}^{2 N-4 j-5} \sin Y_{N+k},
\end{aligned}
$$

for $1 \leq k \leq N$.

Then we get the following result :

Theorem 2.3 The function $v$ defined by:

$$
v(x, y, t)=-2 \frac{\mid \operatorname{det}\left(\left.\left(n_{j k)_{j, k \in[1,2 N]}}\right)\right|^{2}\right.}{\operatorname{det}\left(\left(d_{j k)_{j, k \in[1,2 N]}}\right)^{2}\right.}
$$

is a rational solution to the KPI equation (1).

$$
\left(4 u_{t}-6 u u_{x}+u_{x x x}\right)_{x}-3 u_{y y}=0,
$$

where

$$
\begin{aligned}
& n_{j 1}=\varphi_{j, 1}(x, y, t, 0), 1 \leq j \leq 2 N \quad n_{j k}=\frac{\partial^{2 k-2} \varphi_{j, 1}}{\partial \epsilon^{2 k-2}}(x, y, t, 0), \\
& n_{j N+1}=\varphi_{j, N+1}(x, y, t, 0), 1 \leq j \leq 2 N \quad n_{j N+k}=\frac{\partial^{2 k-2} \varphi_{j, N+1}}{\partial \epsilon^{2 k-2}}(x, y, t, 0), \\
& d_{j 1}=\psi_{j, 1}(x, y, t, 0), 1 \leq j \leq 2 N \quad d_{j k}=\frac{\partial^{2 k-2} \psi_{j, 1}}{\partial \epsilon^{2 k-2}}(x, y, t, 0), \\
& d_{j N+1}=\psi_{j, N+1}(x, y, t, 0), 1 \leq j \leq 2 N \quad d_{j N+k}=\frac{\partial^{2 k-2} \psi_{j, N+1}}{\partial \epsilon^{2 k-2}}(x, y, t, 0), \\
& 2 \leq k \leq N, 1 \leq j \leq 2 N
\end{aligned}
$$

The functions $\varphi$ and $\psi$ are defined in (14),(15), (16), (17).

\section{Explicit Expression of Rational Solutions of Order 5 Depending on 8 Parameters}

In the following, we explicitly construct rational solutions to the KPI equation of order 5 depending on 8 parameters.

Because of the length of the expression, we cannot give it in this text. We only give the expression without parameters in the appendix.

We give patterns of the modulus of the solutions in the plane $(x, y)$ of coordinates in function of parameters $a_{1}, a_{2}, a_{3}, a_{4}, b_{1}, b_{2}, b_{3}, b_{4}$ and time t.

When at least one parameter is not equal to 0 , we observe the presence of 15 peaks. The maximum modulus of these solutions is checked in this case $N=5$, equal to $2(2 N+1)^{2}=2 \times 11^{2}=242$.
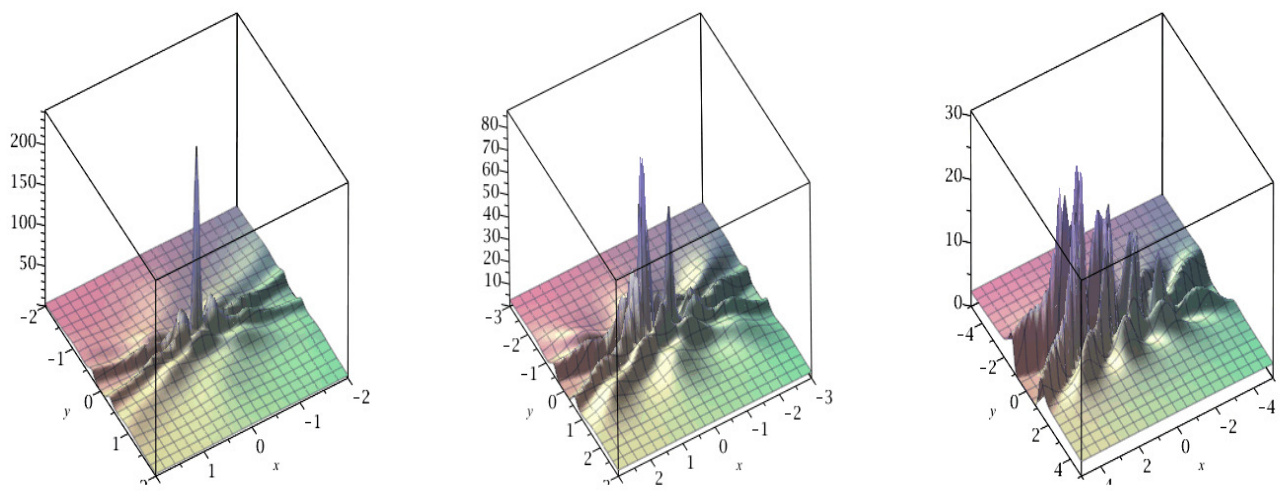

Figure 1. Solution of order 5 to KPI, on the left for $t=0$; in the center for $t=0,01$; on the right for $t=0,1 ;$ all parameters equal to 0. 

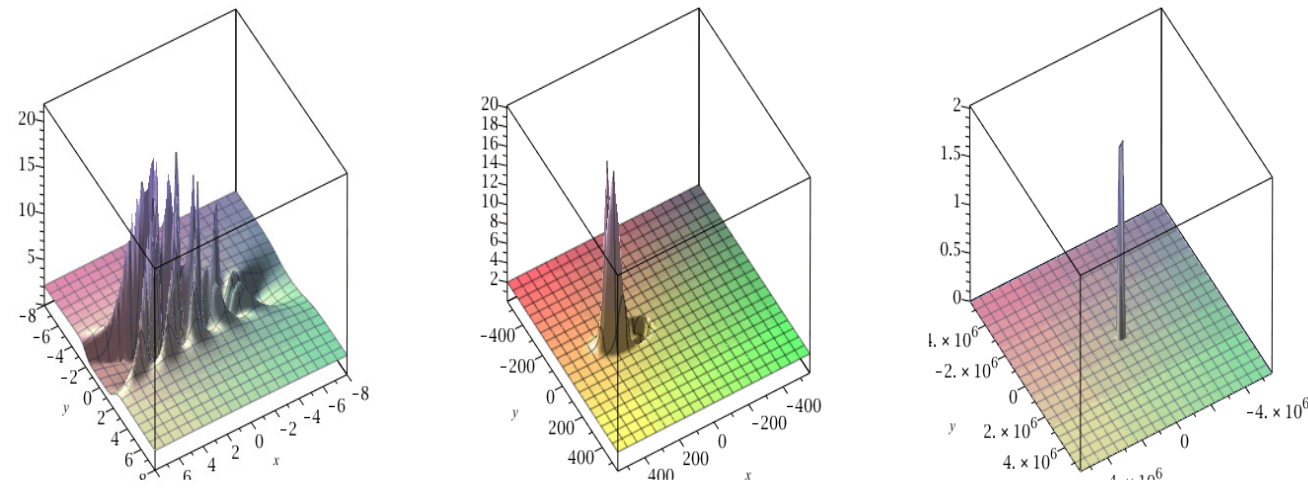

Figure 2. Solution of order 5 to KPI, on the left for $t=0,2$; in the center for $t=20$; on the right for $t=50$; all parameters equal to 0 .
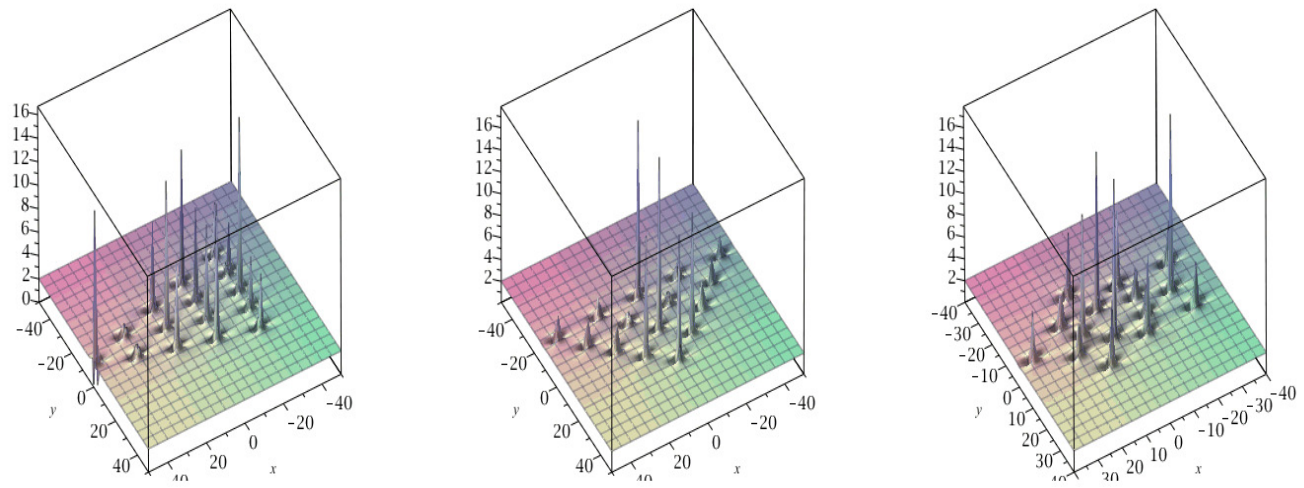

Figure 3. Solution of order 5 to KPI for $t=0$, on the left for $a_{1}=10^{4}$; in the center for $b_{1}=10^{4}$; on the right for $a_{2}=10^{6}$; all other parameters equal to 0 .
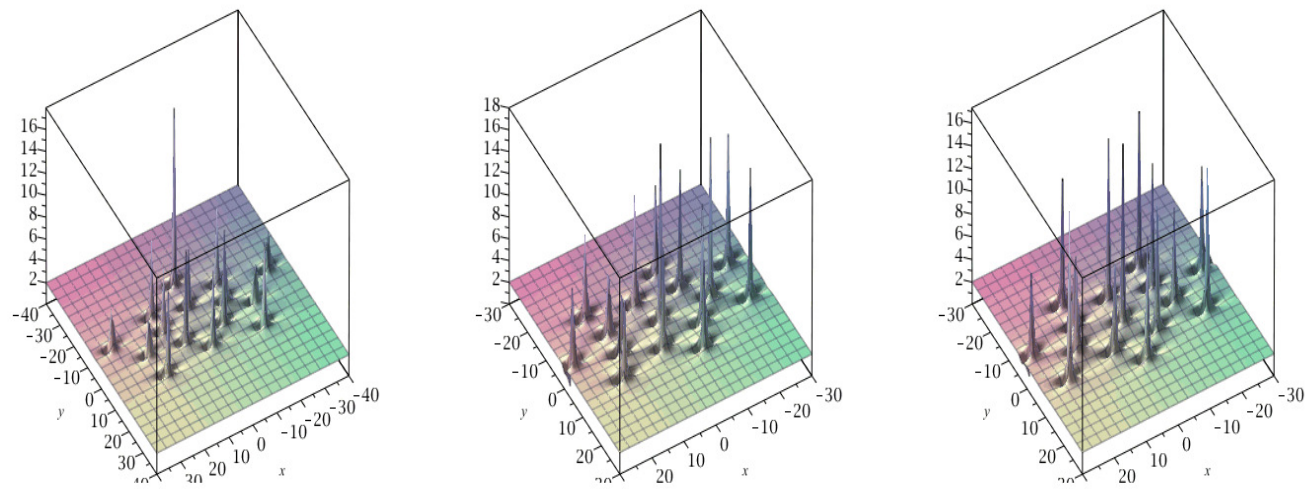

Figure 4. Solution of order 5 to KPI for $t=0$, on the left for $b_{2}=10^{6}$; in the center for $a_{3}=10^{8}$; on the right for $b_{3}=10^{8}$; all other parameters equal to 0 . 

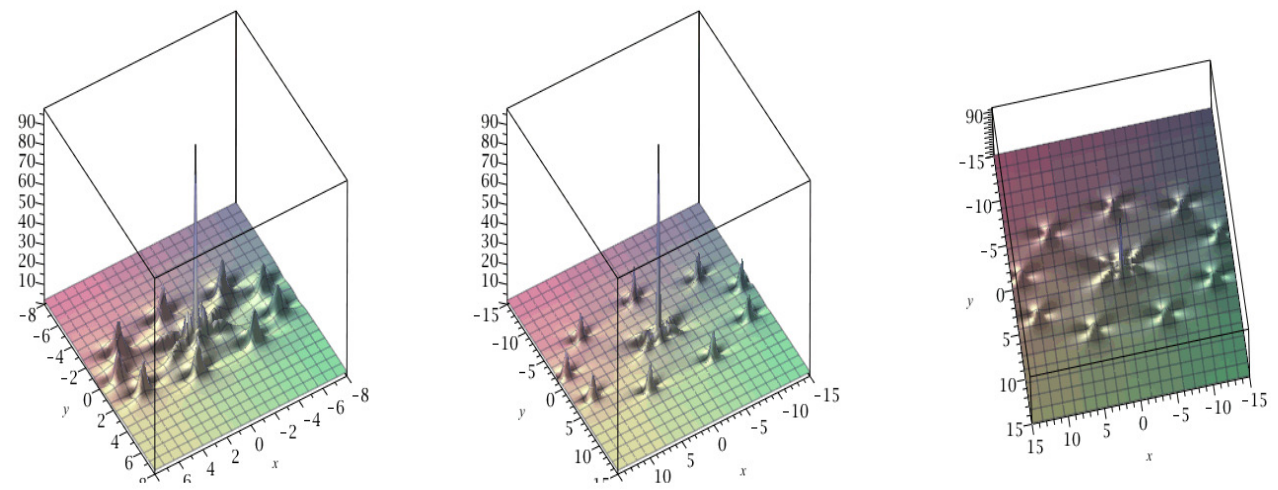

Figure 5. Solution of order 5 to KPI for $t=0$, on the left for $a_{4}=10^{8}$; in the center for $b_{4}=10^{8}$; on the right for $b_{4}=10^{8}$, sight on top; all other parameters equal to 0 .

\section{Conclusion}

We obtain $N$-th order rational solutions to the KPI equation depending on $2 N-2$ real parameters. These solutions can be expressed in terms of a ratio of two polynomials of degree $2 N(N+1)$ in $x, y$ and $t$. The maximum modulus of these solutions is equal to $2(2 N+1)^{2}$. This gives a new approach to find explicit solutions for higher orders and try to describe the structure of these rational solutions. Here, we have given a complete description of rational solutions of order 5 with 8 parameters by giving explicit expressions of polynomials of those solutions.

We construct the modulus of solutions in the $(x, y)$ plane of coordinates; different structures appear. For a given $t$, when one parameter grows and the other ones are equal to 0 we obtain triangular or rings or concentric rings. There are four types of patterns. For $a_{1} \neq 0$ or $b_{1} \neq 0$, and other parameters equal to zero, we obtain a triangle with 15 peaks. For $a_{2} \neq 0$ or $b_{2} \neq 0$, and other parameters equal to zero, we obtain three concentric rings of 5 peaks on each of them. For $a_{3} \neq 0$ or $b_{3} \neq 0$, and other parameters equal to zero, we obtain two concentric rings of 7 peaks on each of them with a central peak; in the last case, when $a_{4} \neq 0$ or $b_{4} \neq 0$, and other parameters equal to zero, we obtain one ring with 9 peaks with the lump $L_{3}$ in the center.

\section{References}

1. B.B. Kadomtsev, V.I. Petviashvili, On the stability of solitary waves in weakly dispersing media, Sov. Phys. Dokl., V. 15, N. 6, 539-541, 1970

2. M.J. Ablowitz, H. Segur On the evolution of packets of water waves, J. Fluid Mech., V. 92, 691-715, 1979

3. D.E. Pelinovsky, Y.A. Stepanyants, Y.A. Kivshar, Self-focusing of plane dark solitons in nonlinear defocusing media, Phys. Rev. E, V. 51, 5016-5026, 1995

4. S.V. Manakov, V.E. Zakharov, L.A. Bordag, V.B. Matveev, Two-dimensional solitons of the KadomtsevPetviashvili equation and their interaction, Phys. Letters, V. 63A, N. 3, 205-206, 1977

5. I. Krichever, Rational solutions of the Kadomtcev-Petviashvili equation and integrable systems of $\mathrm{n}$ particules on a line, Funct. Anal. and Appl., V. 12, N. 1, 59-61, 1978

6. I. Krichever, S.P. Novikov Holomorphic bundles over Riemann surfaces and the KPI equation, Funkt. Ana. E Pril., V. 12, 41-52, 1979

7. J. Satsuma, M.J. Ablowitz, Two-dimensional lumps in nonlinear dispersive systems, J. Math. Phys., V. 20, 1496-1503, 1979

8. V.B. Matveev, Darboux transformation and explicit solutions of the Kadomtcev-Petviaschvily equation depending on functional parameters, Letters in Mathematical Physics, V. 3, 213-216, 1979

9. N. C Freeman, J.J.C. Nimmo Rational solutions of the KdV equation in wronskian form, Phys. Letters, V. 96 A, N. 9, 443-446, 1983 
10. N. C Freeman, J.J.C. Nimmo The use of Bäcklund transformations in obtaining N-soliton solutions in wronskian form, J. Phys. A : Math. Gen., V. 17 A, 1415-1424, 1984

11. D.E. Pelinovsky, Y.A. Stepanyants, New multisolitons of the Kadomtsev-Petviashvili equation, Phys. JETP Lett., V. 57, 24-28, 1993

12. D.E. Pelinovsky, Rational solutions of the Kadomtsev-Petviashvili hierarchy and the dynamics of their poles. I. New form of a general rational solution, J.Math.Phys., V. 35, 5820-5830, 1994

13. M.J Ablowitz, J. Villarroel, Solutions to the time dependent schrödinger and the Kadomtsev-Petviashvili equations, Phys. Rev. Lett., V. 78, 570-573, 1997

14. J. Villarroel, M.J Ablowitz, On the discrete spectrum of the nonstationary Schrdinger equation and multipole lumps of the Kadomtsev-Petviashvili I equation, Commun. Math. Phys., V. 207, 1-42, 1999

15. G. Biondini, Y. Kodama, On a family of solutions of the Kadomtsev-Petviashvili equation which also satisfy the Toda lattice hierarchy, J. Phys. A: Math. Gen., V. 36, 10519-10536, 2003

16. Y. Kodama, Young diagrams and N solitons solutions to the KP equation, J. Phys. A: Math. Gen., V. 37, 11169-11190, 2004

17. G. Biondini, Line Soliton Interactions of the Kadomtsev-Petviashvili Equation, PRL, V. 99, 064103-1-4, 2007

18. P. Gaillard, Families of quasi-rational solutions of the NLS equation and multi-rogue waves, J. Phys. A : Meth. Theor., V. 44, 1-15, 2010

19. P. Gaillard, Degenerate determinant representation of solution of the NLS equation, higher Peregrine breathers and multi-rogue waves, J. Math. Phys., V. 54, 013504-1-32, 2013

20. P. Gaillard, Other $2 \mathrm{~N}-2$ parameters solutions to the NLS equation and $2 \mathrm{~N}+1$ highest amplitude of the modulus of the N-th order AP breather, J. Phys. A: Math. Theor., V. 48, 145203-1-23, 2015

Appendix Because of the length of the complete expression, we only give in this appendix the explicit expression of the rational solution of order 5 to KPI equation without parameters. They can be check via : http://www.isaac-scientific.org/images/template/2017053115412040832.pdf 